\title{
Wide Field Observations of the Ursa Minor dSph Galaxy
}

\author{
D. Martínez-Delgado and A. Aparicio \\ Instituto de Astrofísica de Canarias, 38200 La Laguna, Tenerife, Spain
}

\begin{abstract}
In this paper we present preliminary results of a wide field photometry survey of Ursa Minor and discuss the presence of an intermediate-age population and tidal tails in it.
\end{abstract}

\section{Introduction}

The discovery that the Sagittarius dSph is dissolving into the Galactic halo (Ibata, Gilmore \& Irwin 1994; Mateo et al. 1998) has strengthened the idea that current accretion events could play an important role in the history of the Milky Way formation. Nevertheless, the argument that other dSph satellites can be tidally disrupted by the Milky Way is open to debate. It is thus very important to investigate the structure and history of these dSphs, including stellar populations gradients, traces of recent star formation and the presence of tidal debris in their outer regions. This kind of study using CMDs is very challenging, due to the large angular sizes and low surface density of dSphs, and requires using wide field cameras and a careful analysis of the foreground contamination.

Ursa Minor is one of the closest satellites of the Milky Way $(\mathrm{d}=69 \mathrm{kpc})$. It is possibly a disrupted dSph interacting with the external Galactic halo. This makes its study quite necessary in the aforementioned context. In this paper we present preliminary results of a wide field photometry survey of UMi and discuss the presence of an intermediate-age population and tidal tails in it.

\section{Observations}

The UMi dSph was observed in $B$ and $R$ with the Wide Field Camera (WFC) at the prime focus of the INT $(2.5 \mathrm{~m})$ at the Roque de los Muchachos Observatory (Canary Islands). The WFC holds four $4096 \times 2048$ pixels EEV CCDs with a pixel size of $0.33^{\prime \prime}$, covering a total area of 0.27 square degrees. Figure 1 shows the fields selected for the first observing run of this project.

\section{The Color-Magnitude Diagram of the Ursa Minor dSph}

Figure 2 shows the $[B-R$, " $V$ "] CMDs for the central body of UMi (field A of Fig. 1). These diagrams show a prominent narrow RGB suggesting a small metallicity range in the stellar population. In addition, a well populated, blue extended horizontal branch and several $(\sim 150)$ stars in the RR Lyrae gap region 


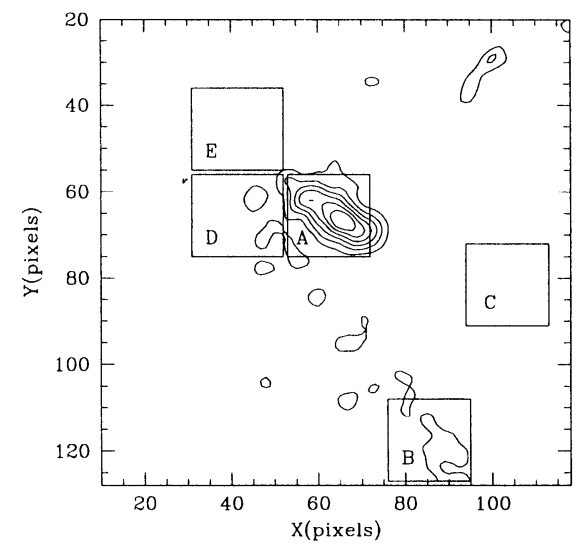

Figure 1. Location of the WFC fields of our study of UMi. North is to the top and East to the left on the isopleth map by Irwin \& Hatzidimitriou (1995). The scale is $1.65^{\prime}$ per pixel.

are observed. The main turn-off corresponds to an age of $\sim 15 \mathrm{Gyr}$ for $Z=$ 0.0004 , based on the Padua stellar evolutionary models (Bertelli et al. 1994, and references therein). The few stars above the subgiant track $(0.6 \leq(B-R) \leq 1.0$; $20.5<$ "V" < 22.5) are probably binary stars although they could be the trace of a not so old population.

Perhaps the most striking feature is the well populated blue plume. A simple explanation is that it would be made of blue stragglers. However, normalized to the RGB, it contains three times more stars than low central density Galactic globular clusters, like NGC 5053 (Nemec \& Cohen 1989). This perhaps indicates that the blue-plume is the trace of an intermediate-age population. Nevertheless, photometry alone is not enough to distinguish blue stragglers from normal main sequence stars. A definitive answer will requires spectroscopic data.

\section{An Intermediate-Age Population in UMi?}

We have done a preliminary estimate of the star formation history for the central body of UMi using the CMD of chip 4 (Fig. 2) and assuming that the blue stars present in the CMDs are not blue-stragglers but an intermediate-age population. The result is shown in Fig. 3 (left panel). A high SFR is required in an early epoch to account for the amount of HB and RGB stars. After $\sim 12$ Gyr ago, a low star formation activity extending until a recent epoch is enough to produce the observed blue-plume. Interestingly, UMi would have maintained this intermediate-age SFR if it somehow could have managed to convert into new stars only $5 \%$ of the material returned to the interstellar medium by older stellar generations.

For illustrative purposes, the right panel of Fig. 3 shows the corresponding synthetic CMD. For simplicity, a constant metallicity $(Z=0.0004)$ has been used and no observational effects have been simulated. 


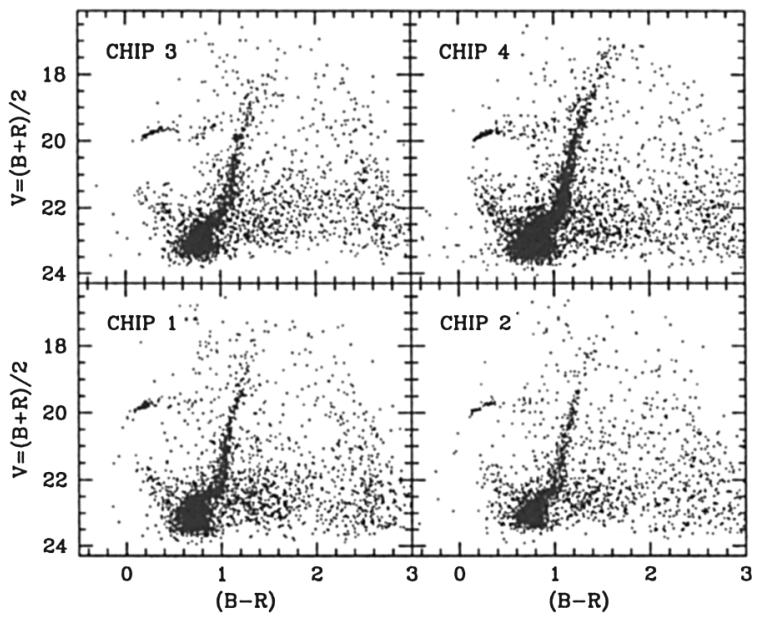

Figure 2. CMDs for the central body of UMi (field A). Each diagram corresponds to one of the four chips of the INT WFC. Chip 4 includes the nucleus of the galaxy.
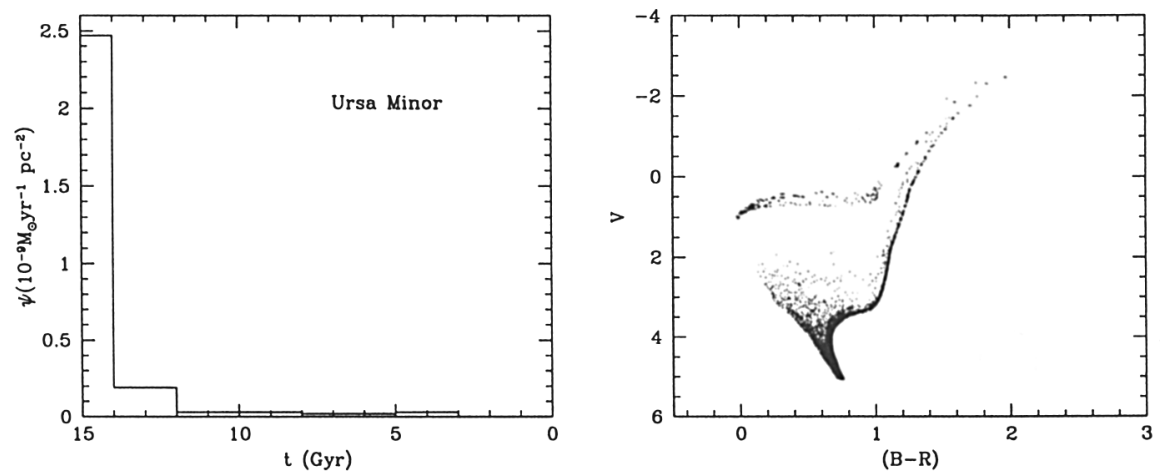

Figure 3. The SFR computed for the central body of UMi (left) and the corresponding synthetic CMD (right). 


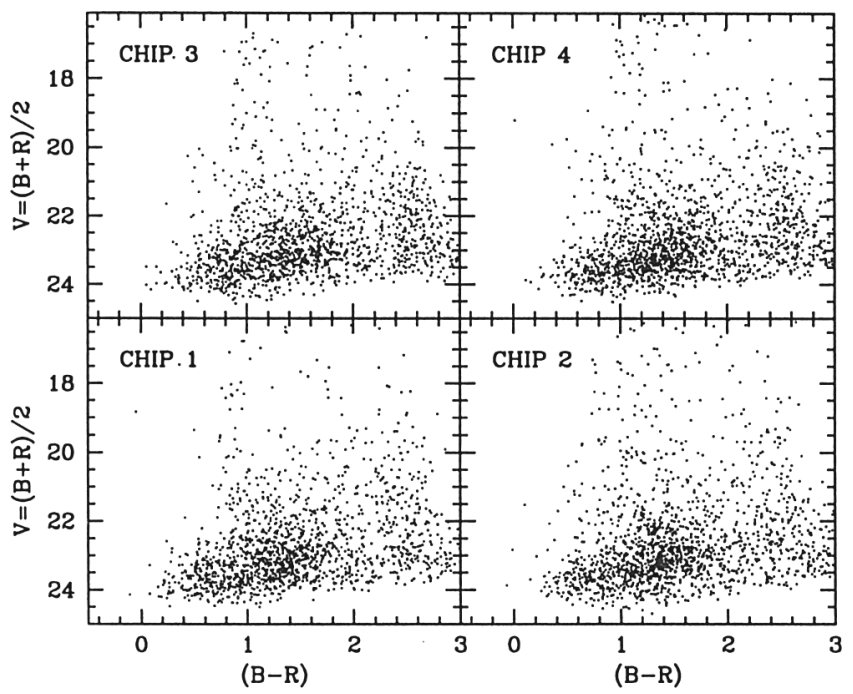

Figure 4. CMDs for the field $\mathrm{C}$ (see Fig. 1) situated in the outer regions of $\mathrm{UMi}$, beyond its tidal radii. No trace of the galaxy is found.

\section{Searching for Tidal Tails}

As a part of our project, we are searching for tidal tails in the periphery of UMi. A first test is the detection of relevant structures in the CMDs of these regions, such as RGB or HB traces. Fig. 4 shows the CMDs of field $\mathrm{C}$ (shown in Fig. 1), situated over the major semiaxis of UMi and beyond its tidal radius in the direction of the Magellanic Stream. No traces of the galaxy are found in this field. A similar result is found for the field B (see Fig. 1). The lack of traces beyond the tidal radius may indicate the absence of extra-tidal stellar debris and suggests that UMi would not be in an advanced state of tidal disruption.

\section{References}

Bertelli, G., Bressan, A., Chiosi, C., Fagotto, F., Nasi, E. 1994, A\&AS, 106, 275

Ibata, R.A., Gilmore, G., Irwin, M.J. 1994, Nature, 370, 194

Irwin, M.J., Hatzidimitriou, D. 1995, MNRAS, 277, 1354

Mateo, M., Olszewski, E.W., Morrison, H.L. 1998, ApJ, in press

Nemec, J.M., Cohen, J.G. 1989, ApJ, 336, 780 